\title{
M-PAM Signals Classification Using Modified Gabor Filter Network
}

\author{
Sajjad Ahmed Ghauri ${ }^{1,2}$ and Ijaz Mansoor Qureshi ${ }^{3}$ \\ ${ }^{1}$ ISRA University, Islamabad, Pakistan \\ ${ }^{2}$ Department of Electronics Engineering, International Islamic University, Islamabad 44000, Pakistan \\ ${ }^{3}$ AIR University, Islamabad, Pakistan \\ Correspondence should be addressed to Sajjad Ahmed Ghauri; sajjad.ghauri@iiu.edu.pk
}

Received 9 September 2014; Revised 2 April 2015; Accepted 15 April 2015

Academic Editor: Angel Sánchez

Copyright (C) 2015 S. A. Ghauri and I. Mansoor Qureshi. This is an open access article distributed under the Creative Commons Attribution License, which permits unrestricted use, distribution, and reproduction in any medium, provided the original work is properly cited.

\begin{abstract}
A Modified Gabor Filter (MGF) network based approach is used for feature extraction and classification of $M$-ary Pulse Amplitude Modulated (M-PAM) signals by adaptively tuning the parameters of MGF network. Modulation classification of $M$-PAM signals is done under the influence of additive white Gaussian noise (AWGN) and channel effects such as Rayleigh flat fading and Rician flat fading. The MGF network uses the network structure of two layers. First layer which is input layer constitutes the adaptive feature extraction part and second layer constitutes the signal classification part. The Gabor atom parameters are tuned using Delta rule and updating of weights of MGF using Recursive Least Square (RLS) algorithm. The simulation results in the form confusion matrix show that proposed modified modulation classification algorithm has high classification accuracy at low signal to noise ratio (SNR). The performance comparison with state-of-the-art existing techniques shows the significant performance improvement of proposed MGF based classifier.
\end{abstract}

\section{Introduction}

Automatic Modulation Classification (AMC) is an approach which classifies the modulation format of the received signal at the receiver side. AMC has found extensive importance in the field of electronic surveillance, military domain, electronic counter measures, civil domain, software defined radios, and lately cognitive radios. For example, in military domains, it may be employed for monitoring and interference recognitions, while in civil domain it includes interference confirmation, spectrum management, and signal confirmation. The most important applications in civil domain are intelligent modems, software defined radios, and cognitive radios. Due to incremental technologies such as cognitive radios, the recent research has been focussed to identify and then classify these types of signal as discussed by Haykin [1].

To accomplish AMC, there are two approaches, decision theoretic approach, which is based upon likelihood function of the received signal, and pattern recognition approach, which is based upon features extraction from the received signal [2]. The likelihood function based decision theoretic approach is optimal, but computationally complex. The classifier based upon decision theoretic approach is proposed in [3]. In [4], author gives survey of the decision theoretic approach and the comparison of proposed classifier performances in the literature. The modulation classification in decision theoretic approach is viewed as multiple hypothesis test or may be sequence of pairwise multiple hypothesis test. Once the likelihood function is set up, average likelihood ratio test (ALRT), generalized likelihood ratio test (GLRT), hybrid likelihood ratio test (HLRT), and combinations of these tests are to be used to determine the modulation format of the received signal [5]. Due to phase errors, channel effects, timing jitter, and frequency offset, the decision theoretic approach is not robust to model mismatch [6]. Maximum likelihood method is used in classification of digital modulations in [7]. The author shows that ML classifier is capable 
of classifying any finite set constellations with zero error rate when the number of available data symbols goes to infinity. The modulation classification algorithm proposed for identification of software defined radio modulation schemes without pilot symbols between transmitter and receiver in [8]. The classifier based upon likelihood ratio test loads the values of test function for likelihood ratio test; the proposed algorithm converts unknown signal symbol to the address of lookup table.

The feature extraction based pattern recognition approach (PRA) is robust to model mismatch, but not optimal with less computational complexity as compared to decision theoretic approach. The PRA is divided into two modules. In the first module, distinct features are extracted from the received signal, which undergo channel effects such as fading and also channel noise such as additive white Gaussian noise (AWGN). After the successful extraction of these features, second module is classifier which decides about the modulation format of the received signal [9].

The previous techniques employed in literature for feature extraction based modulation classification are discussed below. In [10], authors considered seven modulation formats for classification using genetic algorithm (GA) based clustering. The features extracted are spectral features from the received signal and reduced set of parameters is derived from these coefficients and input to GA based clustering technique. The modulation classification based upon combination of 2nd, 4th, 6th, and 8th order cumulants and spectral features are proposed in [11]. Hierarchical support vector machine (SVM) is used as classifier. The optimization Bee algorithm is used to improve the overall performance of proposed classifier. Spectral features, statistical features, and wavelet based features are used to classify the modulation formats in [12] and performance is evaluated on AWGN channel. The authors proposed a classifier based upon SVM and optimization of algorithm is done using particle swarm optimization (PSO). The modulation formats are recognized using artificial neural network (ANN) and resilient back propagation in [13]. The GA is used to select the best feature subset from the combined spectral features and statistical features. The classifier based on a SVM is proposed as multiclass classifier in [14]. The features used are higher order statistics and GA is used for selecting the parameters of classifier. The performance is discussed with or without optimization. The modulation classification of $M$-QAM signals is considered in [15]. The classifier based upon combination of subtractive clustering and PSO is used to extract features. The algorithm gives higher accuracy in the presence of AWGN channel at higher SNRs. Higher order cumulants (HOC) are used as feature set for the classification of several modulation formats in the presence of AWGN channel in [16]. Hybrid classifier which is neural network based is used for classification. The Cramer-Rao lower bound is derived for 4 th order cumulants estimator in [17], and the classification accuracy is measured on AWGN channel. The author proposed a classifier which is based upon optimized distribution sampling test (ODST) for classification [18]. GA is used to optimize distance metrics using sampled distribution parameters. The decision is based upon candidate modulation and distance between tested signals. Time frequency distributions are proposed for modulation classification in [19]. The classification accuracy increased using time frequency features and multilayer classifier are used to classify six modulation formats.

From literature review of the feature based modulation classifications approaches, there are some issues which need to be properly addressed for the development of efficient classifier. The main two issues are choice of extraction of features from the received signal which had undergone channel effects and noise and the classifier structure which is used to discriminate the features for desired modulation format.

In this paper, MGF based efficient features are extracted from the received signal which to the best of our knowledge have not been utilized for the problem of modulation classification of $M$-ary Pulse Amplitude Modulated signals. The features are extracted from the noisy (AWGN) signals plus channel effects (Rayleigh flat fading and Rician flat fading) using MGF network. After successful extraction of the features, weights of adaptive filter are updated using RLS algorithm and classification algorithm efficiently classifies the $M$-PAM signals. Our previous paper for $M$-QAM, $M$-PSK, and $M$-FSK classification was not at all efficient for $M$-PAM signals [20]. In this paper, we have made two important changes to make it efficient for M-PAM signals. The classification accuracy of the proposed classifier is also compared with well-known state-of-the-art existing techniques.

The rest of the paper is organized as follows. Section 2 represents the Modified Gabor Filter network and system model and also feature extraction using Gabor filter is presented. In Section 3, Modified Gabor Filter algorithm for training and testing is presented. In Section 4, performance of proposed modified classifier in the presence of AWGN channel, Rician fading channel, and Rayleigh fading channel is presented. Section 5 concludes the paper.

\section{Gabor Filter Based System Model}

2.1. System Model. The generalized expression for signal received is given by a work of Ghauri et al. [2]:

$$
r(n)=s(n)+g(n),
$$

where $r(n)$ is complex baseband envelope of received signal and $g(n)$ is the additive white Gaussian noise with zero mean and a variance of $\sigma_{g}^{2}$. The $s(n)$ is defined as

$$
s(n)=A e^{i\left(w_{o} n T+\theta_{n}\right)} \sum_{j=-\infty}^{\infty} s(l) h\left(n \tau-j \tau+\epsilon_{T} \tau\right),
$$

where $s(l)$ is the input symbol sequence which is drawn from set of $M$ constellations of known symbols and it is not necessary that symbols are equiprobable. $A$ is the amplitude of signal, $w_{o}$ is angular frequency offset constant, $\tau$ is symbol spacing, $\theta_{n}$ is the phase jitter which varies from symbol to symbol, $h(\cdot)$ is the channel effects, and $\epsilon_{T}$ is the timing jitter. The system model for classification of $M$-PAM signals is shown in Figure 1. The received signal is first preprocessed in which the main part is to remove the noise from the signal 


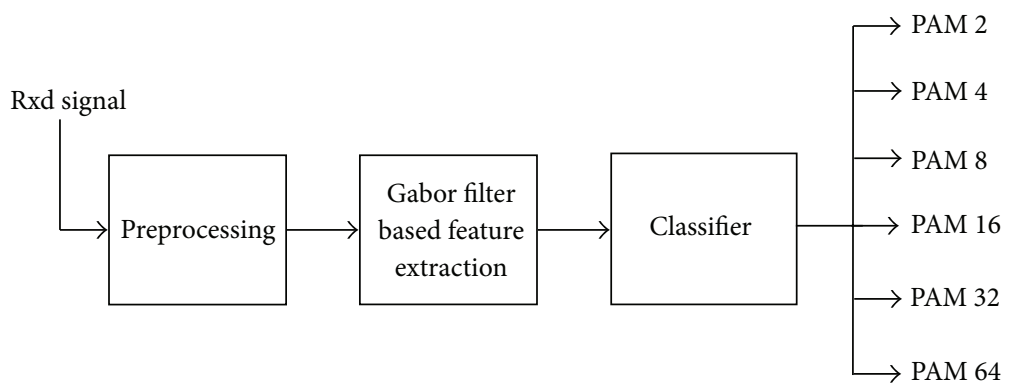

FIGURE 1: System model for classification of $M$-PAM signals.

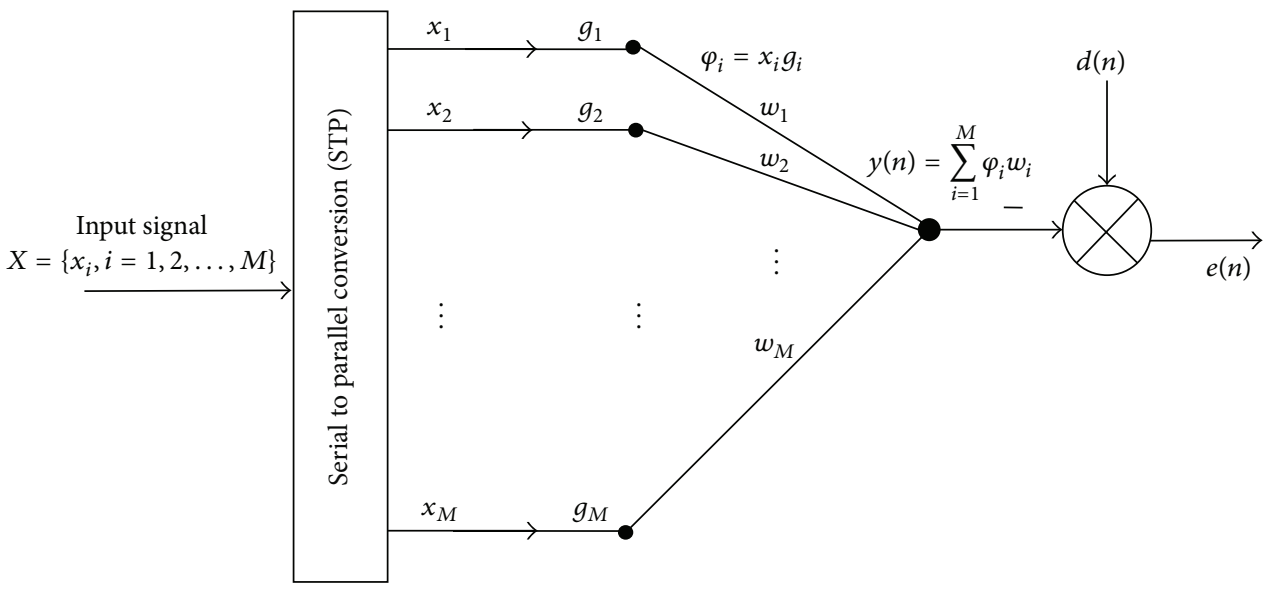

Figure 2: Training of Modified Gabor Filter network for M-PAM signals classification.

or to eliminate the sources of variations. After preprocessing efficient features are extracted using Gabor filter network and these features are used for classification of $M$-PAM formats among class of $M$-PAM signals.

\subsection{Feature Extraction Using Modified Gabor Filter Network.} The Gabor atom is used for the extraction of features and in generalized form it can be written as

$$
g_{(c, \sigma, f)}(t)=\frac{1}{\sqrt{\sigma}} g\left(\frac{t-c}{\sigma}\right) e^{j f t}
$$

where $g(t)=2^{1 / 4} e^{-\pi t^{2}}$ and $c, \sigma$, and $f$ are shift parameter, scale parameter, and modulation parameter, respectively [20]. There are two layer structures for Gabor filter; in first layer, features are extracted adjusting the Gabor atom parameters $(c, \sigma, f)$ until some cost function is minimized. In second layer, adjustment of adaptive filter weights and the classification process is to be done. As seen from Figure 2, $X=\left\{x_{i}, i=1,2, \ldots, M\right\}$ input to the filter is first serial to parallel converted and after that Gabor atom nodes are calculated using the relationship $\varphi_{i}=\left|\left\langle g_{i}, x_{i}\right\rangle\right|$. The output of the $i$ th Gabor atom node is $\varphi_{i}$ corresponding to input signal $x_{i}$. Thus, output of Gabor atom is defined as

$$
\phi(i)=\left|\int \frac{1}{\sqrt{\sigma_{i}}} g^{*}\left(\frac{t-c_{i}}{\sigma_{i}}\right) e^{-j f_{i} t} x_{i}(t) d t\right| .
$$

The Gabor atom $\left\{g_{i}, i=1,2,3, \ldots, M\right\}$ is defined as

$$
g(i, t)=\frac{1}{\sqrt{\sigma_{i}}} g\left(\frac{t-c_{i}}{\sigma_{i}}\right) e^{j f_{i} t} .
$$

The output of the Gabor atom node $\varphi_{i}$ in the input layer is weighted by $w_{i}$; that is,

$$
y(n)=\sum_{i=1}^{M} \varphi_{i} w_{i}
$$

where $n=1,2, \ldots, N$. The difference between the desired outputs $d(n)$ and actual output $y(n)$ is defined as

$$
e(n)=d(n)-y(n) .
$$

The cost function is square of error function $e(n)$ which is given by

$$
J(n)=[d(n)-y(n)]^{2} .
$$

The four parameters of Gabor filter network and adaptive filter $(c, \sigma, f, w)$ are adjusted until the $J(n)$ is minimized and approaches to zero.

\section{Testing and Training of Proposed Algorithm}

To classify the $M$-PAM signals, the training and testing of the proposed algorithm have to be done. The PAM formats are 
spread about axis, and as increasing the $M$ which may vary from 2 to 64, the values of amplitudes are also increasing. The increased values of amplitudes destroy the convergence of the algorithm. To cope up with the problem of divergence, following are the proposed changes in the existing algorithm [20] for classification of PAM formats:

(1) The absolute values of amplitude are taken instead of taking whole input modulated signal; for example, PAM 4 have amplitudes $\{-3,-1,1,3\}$ but only take absolute values of amplitudes, that is, $\{3,1,1,3\}$.

(2) The desired responses for each of the considered modulation formats are the average amplitudes

$$
a=\sum_{j=1}^{M}\left(\frac{\left|A_{j}\right|}{M}\right)
$$

where $\left\{A_{j} \varepsilon-7,-5,-3,-1,1,3,5,7\right\}$, for example, for PAM 8, and the desired response for the PAM 8 is 4 .

(3) The weights of the adaptive filter are updated using Recursive Least Square (RLS) algorithm instead of using Least Mean Square (LMS) algorithm for the two motives. First, the convergence rate of RLS is sooner than the LMS. Second, the mean square error produced by RLS is lesser than the LMS.

(A) Training of MGF Network. The training of MGF network for PAM 2, PAM 4, PAM 8, PAM 16, PAM 32, and PAM 64 is carried out by adjusting the three parameters of MGF network which are shift, scale, and modulation parameters $(c, \sigma, f)$ and weights of the adaptive filter $(w)$. Figure 2 shows the training of Gabor filter network by adjusting the three parameters and weights of the adaptive filter by using Delta rule and RLS algorithm, respectively. The training process continues until error function is minimized to some threshold or approaches to zero.

To update $(c, \sigma, f)$, Delta rule is used to calculate the change in shift parameter $c_{i}$, scale parameter $\sigma_{i}$, and modulation parameter $f_{i}$ :

$$
\begin{aligned}
\Delta c_{i} & =c_{i}(n+1)-c_{i}(n), \\
\Delta c_{i} & =-\frac{\eta_{c}}{2}\left[\frac{\partial J(n)}{\partial \varphi_{i}} \frac{\partial \varphi_{i}}{\partial c_{i}}\right], \\
\Delta \sigma_{i} & =\sigma_{i}(n+1)-\sigma_{i}(n), \\
\Delta \sigma_{i} & =-\frac{\eta_{\sigma}}{2}\left[\frac{\partial J(n)}{\partial \varphi_{i}} \frac{\partial \varphi_{i}}{\partial \sigma_{i}}\right], \\
\Delta f_{i} & =f_{i}(n+1)-f_{i}(n), \\
\Delta f_{i} & =-\frac{\eta_{f}}{2}\left[\frac{\partial J(n)}{\partial \varphi_{i}} \frac{\partial \varphi_{i}}{\partial f_{i}}\right] .
\end{aligned}
$$

To minimize the cost function $J(n)$ for the Gabor filter network parameters $(c, \sigma, f)$, we take the partial derivatives with respect to shift parameter $c_{i}$, scale parameter $\sigma_{i}$, and modulation parameter $f_{i}$ :

$$
\frac{\partial J(n)}{\partial \varphi_{i}}=-2[d(n)-y(n)] \frac{\partial}{\partial \varphi_{i}} y(n) .
$$

From (6),

$$
\frac{\partial}{\partial \varphi_{i}} y(n)=w_{i}
$$

Using the above result in (11),

$$
\frac{\partial J(n)}{\partial \varphi_{i}}=-2[d(n)-y(n)] w_{i}
$$

From (4),

$$
\phi_{i}=\left|x_{i} \frac{1}{\sqrt{\sigma_{i}}} e^{-\pi\left(\left(t-c_{i}\right) / \sigma_{i}\right)^{2}} \cos \left(f_{i} t\right)\right| .
$$

The partial derivatives of $\varphi_{i}$ with respect to shift parameter $c_{i}$, scale parameter $\sigma_{i}$, and modulation parameter $f_{i}$ are as follows [20]:

$$
\begin{aligned}
\frac{\partial \varphi_{i}}{\partial c_{i}} & =\frac{\partial}{\partial c_{i}}\left(x_{i} g_{i}\right) \\
& =\frac{x_{i}}{\sqrt[5]{\sigma_{i}}} \cos \left(f_{i} t\right) 2 \pi\left(t-c_{i}\right) e^{-\pi\left(\left(t-c_{i}\right) / \sigma_{i}\right)^{2}}, \\
\frac{\partial \varphi_{i}}{\partial \sigma_{i}} & =\frac{\partial}{\partial \sigma_{i}}\left[x_{i} \frac{1}{\sqrt{\sigma_{i}}} e^{-\pi\left(\left(t-c_{i}\right) / \sigma_{i}\right)^{2}} \cos \left(f_{i} t\right)\right] \\
& =\frac{x_{i} \cos \left(f_{i} t\right)}{\sqrt{\sigma_{i}}} e^{-\pi\left(\left(t-c_{i}\right) / \sigma_{i}\right)^{2}}\left[\frac{2 \pi\left(t-c_{i}\right)^{2}}{\sigma_{i}^{3}}-\frac{1}{2 \sigma_{i}}\right], \\
\frac{\partial \varphi_{i}}{\partial f_{i}} & =\frac{\partial}{\partial f_{i}}\left[x_{i} \frac{1}{\sqrt{\sigma_{i}}} e^{-\pi\left(\left(t-c_{i}\right) / \sigma_{i}\right)^{2}} \cos \left(f_{i} t\right)\right] \\
& =-\frac{t}{\sqrt{\sigma_{i}}} x_{i} e^{-\pi\left(\left(t-c_{i}\right) / \sigma_{i}\right)^{2}} \sin \left(f_{i} t\right) .
\end{aligned}
$$

The updated Gabor filter parameters are as follows [20]:

$$
\begin{aligned}
& c_{i}(n+1)=c_{i}(n)+\left[\eta_{c}\{d(n)-y(n)\} w_{i}\right] \\
& \cdot\left[\frac{x_{i}}{\left.\sqrt[5]{\sigma_{i}} \cos \left(f_{i} t\right) 2 \pi\left(t-c_{i}\right) e^{-\pi\left(\left(t-c_{i}\right) / \sigma_{i}\right)^{2}}\right],}\right. \\
& \sigma_{i}(n+1)=\sigma_{i}(n)+\left[\eta_{\sigma}\{d(n)-y(n)\} w_{i}\right] \\
& \cdot\left\{\frac{x_{i} \cos \left(f_{i} t\right)}{\sqrt{\sigma_{i}}} e^{-\pi\left(\left(t-c_{i}\right) / \sigma_{i}\right)^{2}}\left[\frac{2 \pi\left(t-c_{i}\right)^{2}}{\sigma_{i}^{3}}-\frac{1}{2 \sigma_{i}}\right]\right\}, \\
& f_{i}(n+1)=f_{i}(n)+\left[\eta_{f}\{d(n)-y(n)\} w_{i}\right] \\
& \cdot\left\{-\frac{t}{\sqrt{\sigma_{i}}} x_{i} e^{-\pi\left(\left(t-c_{i}\right) / \sigma_{i}\right)^{2}} \sin \left(f_{i} t\right)\right\} .
\end{aligned}
$$




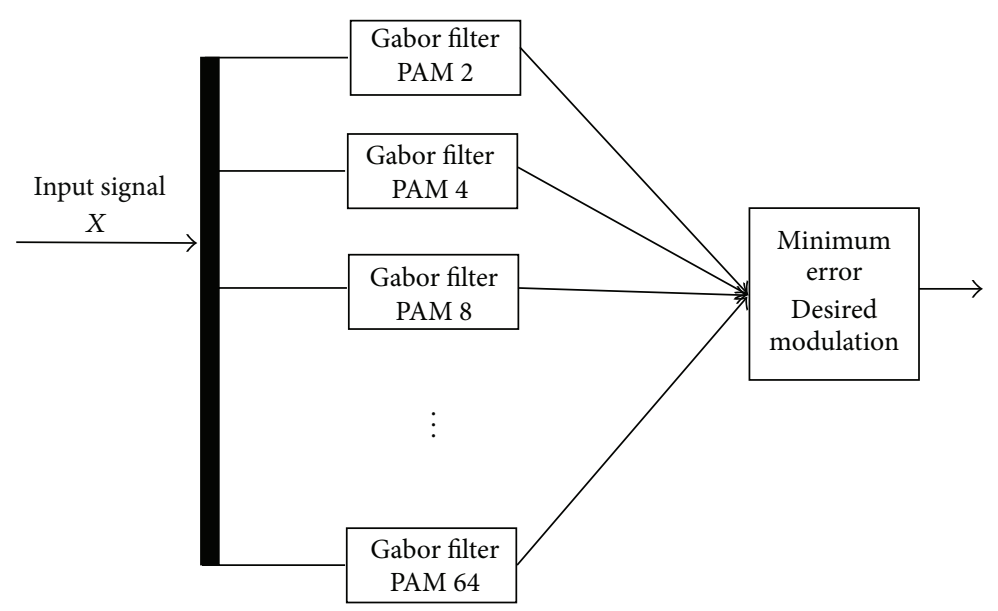

FIGURE 3: Testing of Modified Gabor Filter network for $M$-PAM signals classification.

The weights of adaptive filter are updated using RLS algorithm as follows:

$$
\begin{aligned}
& k(n)=\frac{K(n-1) \varphi(n)}{\lambda+\varphi^{T}(n) K(n-1) \varphi(n)}, \\
& e(n)=d(n)-y(n)=d(n)-\sum_{i=1}^{M} \varphi_{i} w_{i}, \\
& w(n)=w(n-1)+k(n) e(n), \\
& K(n)=\lambda^{(-1)} K(n-1)-\lambda^{(-1)} k(n) \varphi^{T}(n) K(n-1) .
\end{aligned}
$$

To initialize the algorithm, weights are initialized as $w(0)=$ $[1,1, \ldots, 1]$ and the $K$ is referred to as inverse correlation matrix. The $\varphi(n)$ is the input vector and $\lambda$ is forgetting factor.

Algorithm 1 (training of Modified Gabor Filter network for modulation classification).

Step 1. Initialize Gabor atom parameters.

Step 2. Compute all Gabor atom nodes using (14).

Step 3. Adjust adaptive filter using RLS (17).

Step 4. After adjusting the weights, calculate error form (7).

Step 5. If error is less than chosen threshold, then training of algorithm is stopped and save Gabor atom parameters $\left(c_{i}, \sigma_{i}, f_{i}\right)$ and Gabor filter weights $w_{i}$.

Step 6. If error is not less than threshold, repeat step (3) by using the error calculated in step (4).

Step 7. Tune the Gabor atom parameters $\left(c_{i}, \sigma_{i}, f_{i}\right)$ using (16).

(B) Testing of MGF Network. Figure 3 shows the testing of MGF network by computing the error function of each Gabor filter network. The minimum error corresponds to the desired modulation format among class of $M$-PAM signals.
The algorithm for testing of MGF network for classification of $M$-PAM signals is as shown below.

Algorithm 2 (testing of Modified Gabor Filter network for modulation classification).

Step 1. Input digital modulated signal which may be PAM 2 to 64 modulated.

Step 2. Compute the output of each Gabor filter network by using the relation

$$
y=\sum_{i=1}^{M} \varphi_{i} w_{i} .
$$

Step 3. Compute the error function of each Gabor filter network.

Step 4. Minimum error corresponds to the desired modulation format of the input signal.

\section{Simulation Results}

The simulation results are divided into two modules; in the first module, training of Gabor filter network is evaluated for the $M$-PAM signal classification in tabular form and also curves for mean square error versus number of iterations and signal to noise ratio are evaluated. The considered modulation formats are trained accordingly in the class of $M$-PAM signals. The received signal is also corrupted form AWGN and efficient features are calculated and used for training of Gabor filter network. At the end of training, the three parameters of Gabor filter network (shift, scale, and modulation parameters) and adaptive filter weights are saved for minimum mean square error. In the second module, the testing of Gabor filter network is carried out by finding the error function of each Gabor filter network and minimum error corresponds to desired modulation format. The simulation results in testing module are in the form of probability of correctness curve versus SNR under the effects of AWGN and channel effects. 


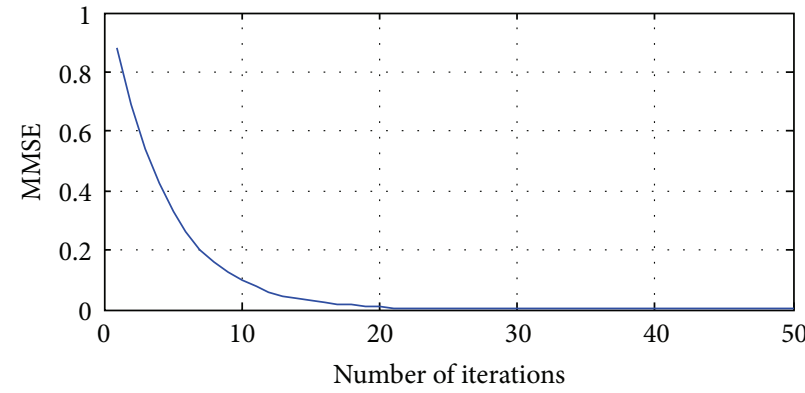

- PAM 2

(a)

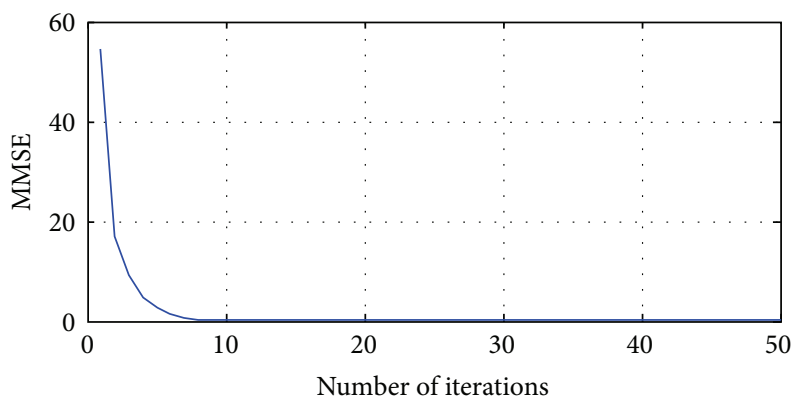

- PAM 8

(c)

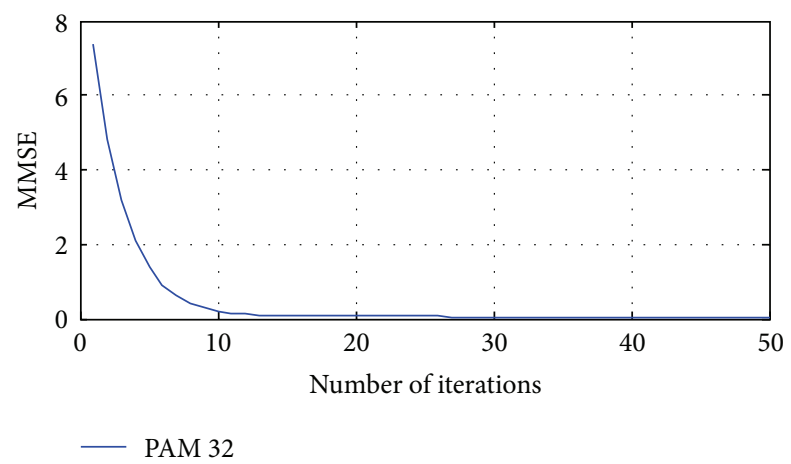

(e)

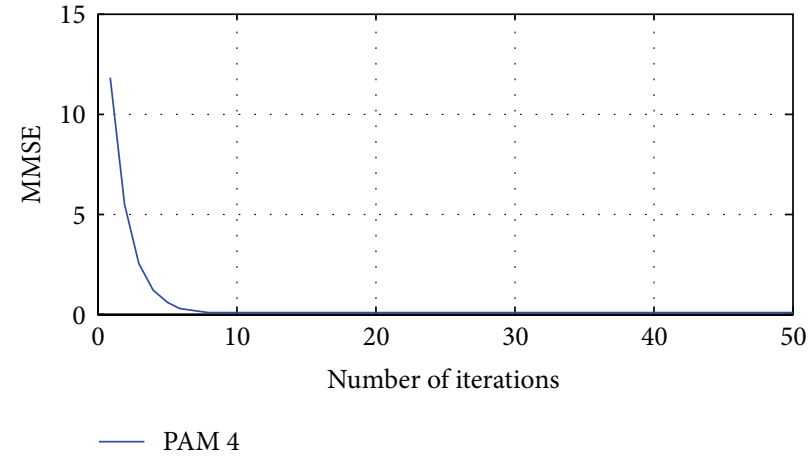

(b)

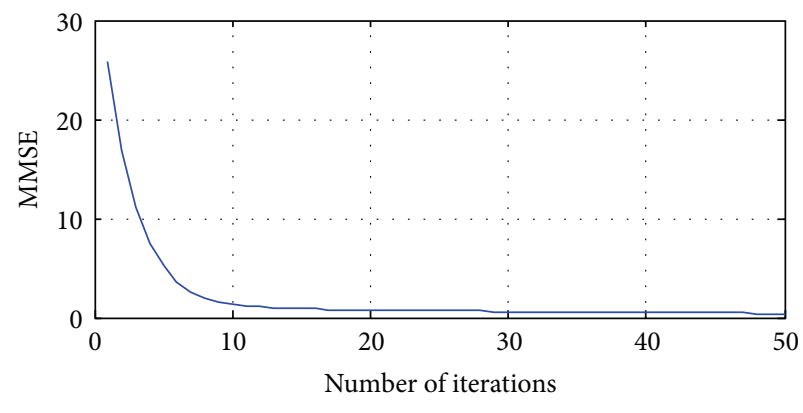

- PAM 16

(d)

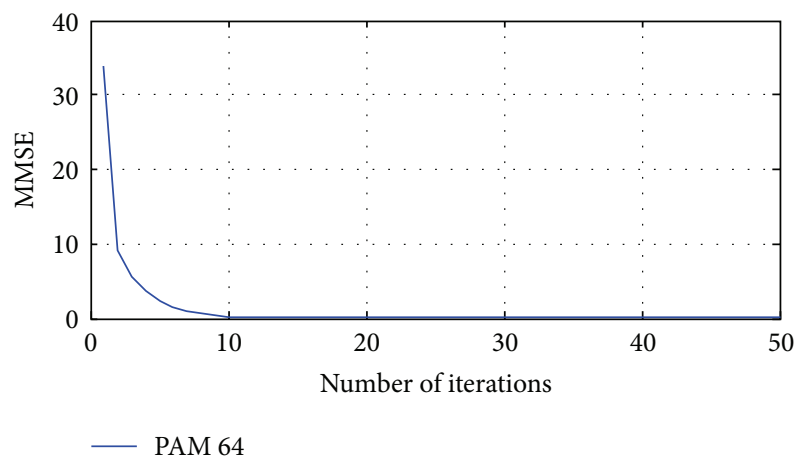

(f)

FIGURE 4: Training of Gabor filter network for the M-PAM formats under no noise.

4.1. Training of Modified Gabor Filter Network. Figure 4 shows the training of Gabor filter network under no noise conditions. From Figure 4, it is clear that the minimum means square error (MMSE) is approaching to zero as the number of iterations increases for all considered modulation formats. The training of network is stopped when MMSE reaches some threshold or zero and the features are stored.

Figure 5 shows the training of Gabor filter network under the influence of AWGN channel with fixed number of iterations. The MMSE is approaching to zero as SNR increases from -10 to $20 \mathrm{~dB}$ for the considered modulation formats as shown in Figure 5.

Table 1 shows the training performance of Gabor filter network in the form of diagonal matrix or accuracy matrix for the classification of considered modulation formats
TABLE 1: Training performance of Gabor filter network of $M$-PAM signal classification without noise.

\begin{tabular}{lcccccc}
\hline & PAM 2 & PAM 4 & PAM 8 & PAM 16 & PAM 32 & PAM 64 \\
\hline PAM 2 & $100 \%$ & & & & & \\
PAM 4 & & $100 \%$ & & & & \\
PAM 8 & & & $100 \%$ & & & \\
PAM 16 & & & & $99.2 \%$ & & \\
PAM 32 & & & & & $99.6 \%$ & \\
PAM 64 & & & & & $100 \%$ \\
\hline
\end{tabular}

(PAM 2, PAM 4, PAM 8, PAM 16, PAM 32, and PAM 64). The training performance is approximately $100 \%$ under no noise considerations. 


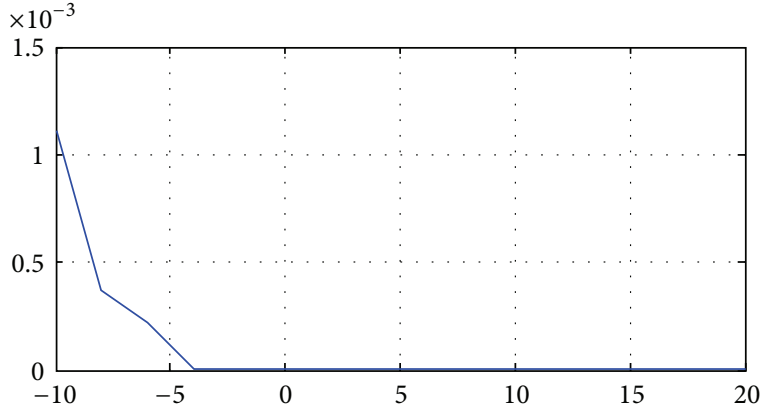

- PAM 2

(a)

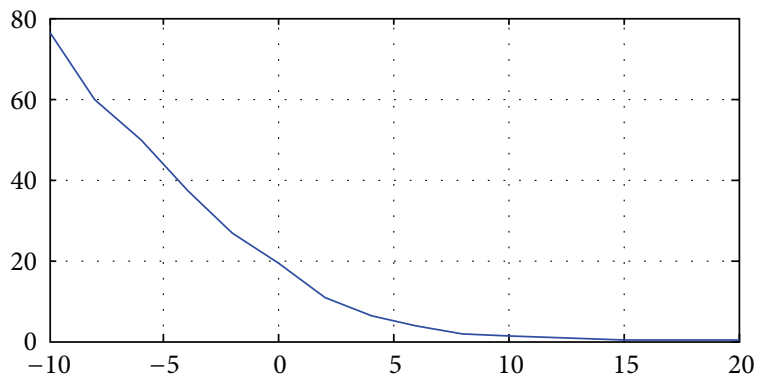

- PAM 8

(c)

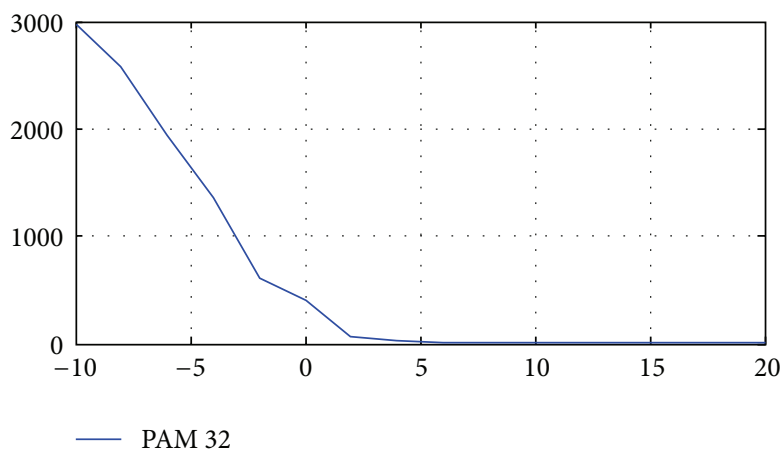

(e)

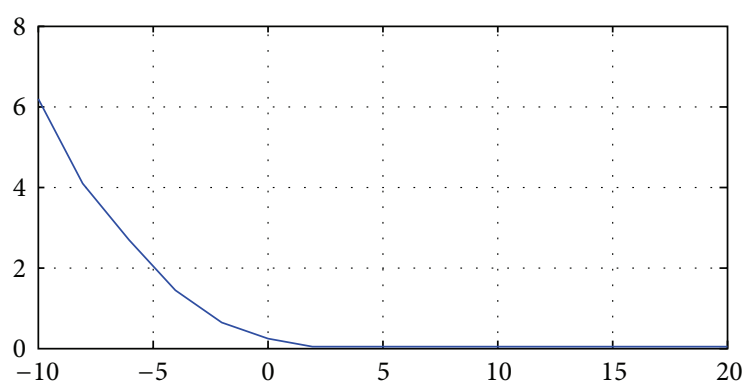

PAM 4

(b)

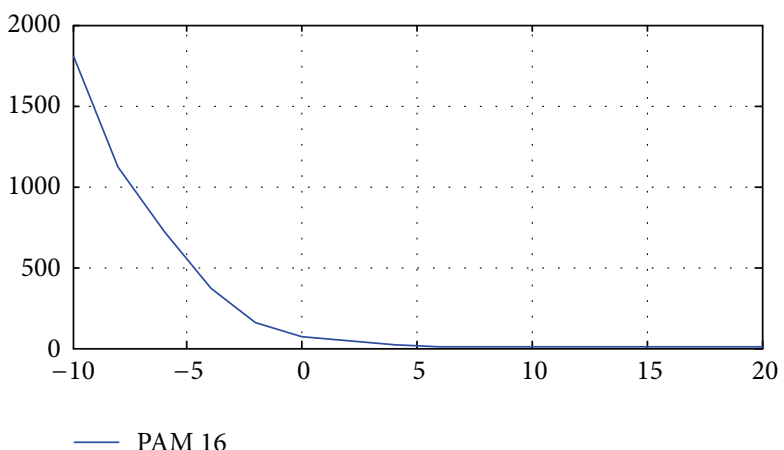

(d)

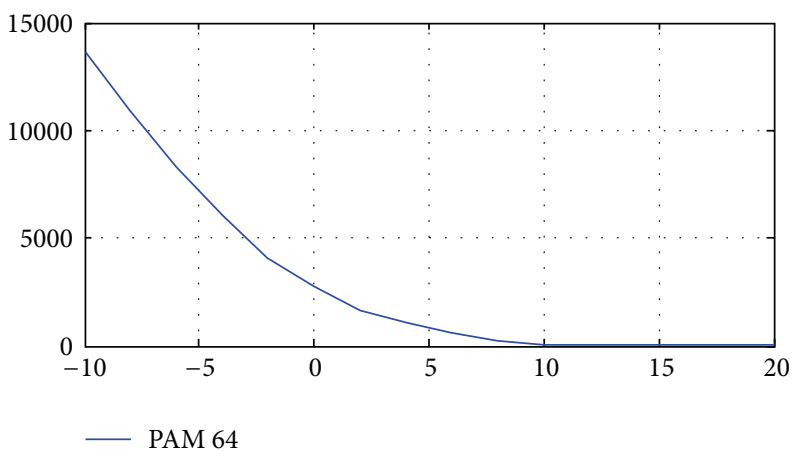

(f)

FIGURE 5: Training of Gabor filter network for the M-PAM formats on AWGN channel.

TABLE 2: Training performance of Gabor filter network of $M$-PAM signal classification on AWGN channel.

\begin{tabular}{lcccccc}
\hline & PAM 2 & PAM 4 & PAM 8 & PAM 16 & PAM 32 & PAM 64 \\
\hline PAM 2 & $100 \%$ & & & & & \\
PAM 4 & & $99.9 \%$ & & & & \\
PAM 8 & & $100 \%$ & & & \\
PAM 16 & & & $98.4 \%$ & & \\
PAM 32 & & & & $99.2 \%$ & \\
PAM 64 & & & & & $99.3 \%$ \\
\hline
\end{tabular}

Table 2 shows the training performance of Gabor filter network in the form of diagonal matrix or accuracy matrix for the classification of $M$-PAM signals under the influence of additive white Gaussian noise. The training of Gabor filter network is done at SNR of $5 \mathrm{~dB}$ and accuracy is approximately 99.5\% for considered modulation formats.

4.2. Testing of Modified Gabor Filter Network. Table 3 shows the testing performance of Gabor filter network in the form of diagonal matrix for the $M$-PAM signal classification. The performance is evaluated at SNR of $5 \mathrm{~dB}$ and it is shown from the table that percentage accuracy for classifying the modulation formats is much better at low SNRs.

Table 4 shows the testing performance of Gabor filter network in the form of diagonal matrix for the $M$-PAM signal classification at SNR of $10 \mathrm{~dB}$ and it is shown from the table that percentage accuracy for classifying the modulation 
TABLE 3: Testing performance of Gabor filter network of $M$-PAM signal classification at $\mathrm{SNR}=5 \mathrm{~dB}$ on AWGN channel.

\begin{tabular}{lcccccc}
\hline & PAM 2 & PAM 4 & PAM 8 & PAM 16 & PAM 32 & PAM 64 \\
\hline PAM 2 & $98.6 \%$ & & & & & \\
PAM 4 & & $97.8 \%$ & & & & \\
PAM 8 & & $96.6 \%$ & & & \\
PAM 16 & & & $96.1 \%$ & & \\
PAM 32 & & & & $98.1 \%$ & \\
PAM 64 & & & & & $97.6 \%$ \\
\hline
\end{tabular}

TABLE 4: Testing performance of Gabor filter network for M-PAM signal classification at $\mathrm{SNR}=10 \mathrm{~dB}$ on AWGN channel.

\begin{tabular}{lcccccc}
\hline & PAM 2 & PAM 4 & PAM 8 & PAM 16 & PAM 32 & PAM 64 \\
\hline PAM 2 & $99.5 \%$ & & & & & \\
PAM 4 & & $98.9 \%$ & & & & \\
PAM 8 & & $99.9 \%$ & & & \\
PAM 16 & & & $98.3 \%$ & & \\
PAM 32 & & & & $98.5 \%$ & \\
PAM 64 & & & & & $98.9 \%$ \\
\hline
\end{tabular}

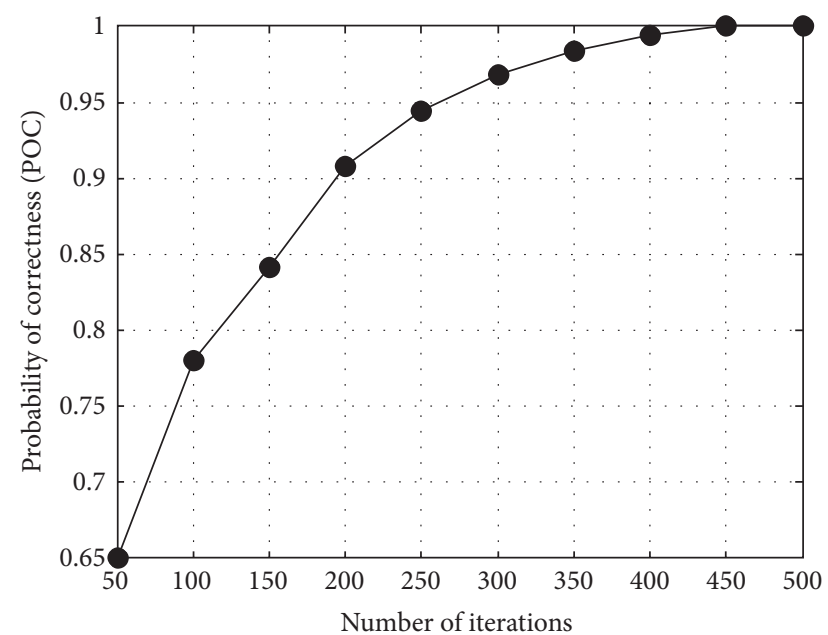

FIgURE 6: Probability of correctness curve for the example of PAM 16.

formats is $98.7 \%$. The testing performance is better due to two facts: first choice of efficient features from the Gabor filter network and second the classifier.

Figure 6 shows the probability of correctness (POC) plotted against number of iterations and from Figure 6, it is clear that POC is approximately 1 when number of iterations increased up to 500. The example considered in Figure 6 is PAM 16 among class of $M$-PAM signals which are classified correctly.

Figure 7 shows the probability of correctness (POC) plotted against signal to noise (SNR) for fixed number of iterations and from Figure 7 it is clear that POC is approximately 1 when SNR is increased up to 10 . The example considered in Figure 7 is PAM 16 and classification accuracy is approximately $90 \%$ at $\mathrm{SNR}$ greater than $3 \mathrm{~dB}$.

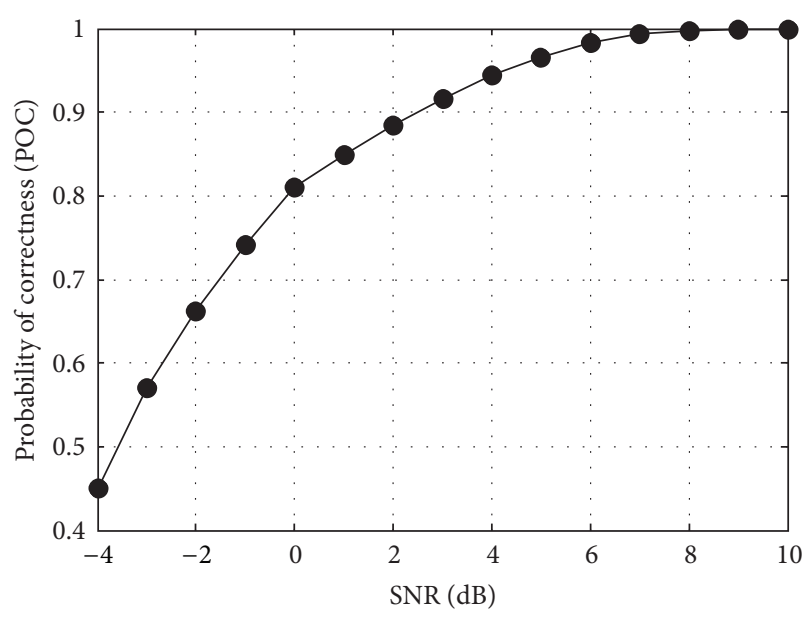

FIGURE 7: Probability of correctness curve under AWGN channel for the example of PAM 16.

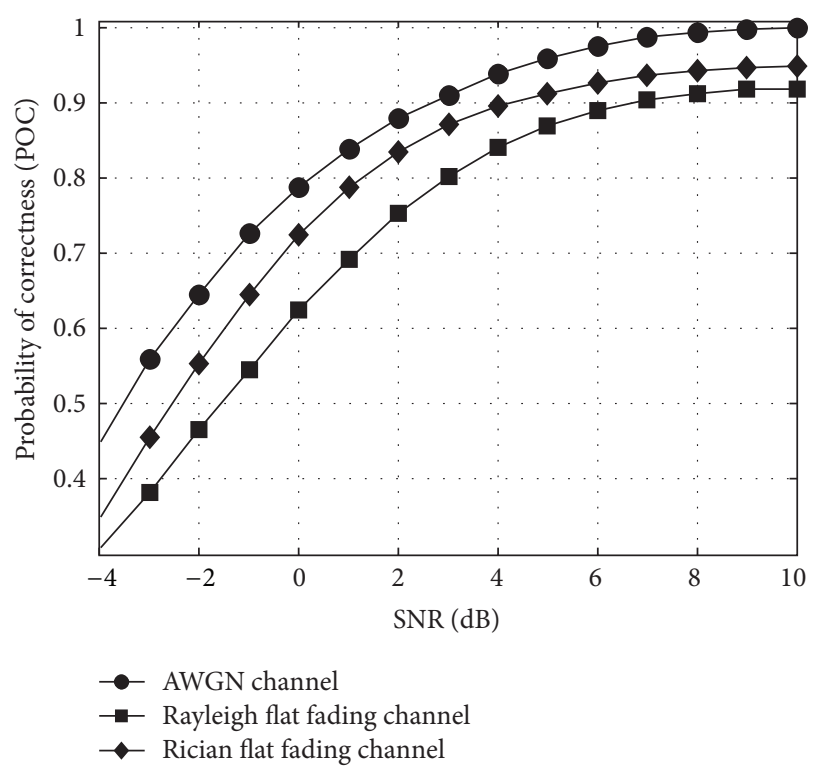

FIGURE 8: Performance comparison under the effect of AWGN and faded channel for the example of PAM 8.

The classification performance of PAM 8 considered example among the $M$-PAM signals is evaluated in Figure 8. Figure 8 also shows the performance comparison under the effect of AWGN channel, Rician flat fading channel, and Rayleigh flat fading channel. The classification accuracy is much better for the considered example on considered channels.

Table 5 shows the performance comparison of percentage classification on the AWGN channel, Rician flat fading channel, and Rayleigh flat fading channel for the example of 8-PAM among class of $M$-PAM signals. The classifier performance is evaluated for $\mathrm{SNR}-4$ to $10 \mathrm{~dB}$ on fading channel plus AWGN. The classifier performance is approximately $100 \%$ on AWGN channel, $95 \%$ on Rician flat fading channel, and $92 \%$ for the Rayleigh flat fading channel at SNR of $10 \mathrm{~dB}$. 
TABLE 5: Testing performance comparison of Gabor filter network on AWGN and fading channels for the example of 8-PAM format.

\begin{tabular}{lccc}
\hline SNR in $\mathrm{dB}$ & AWGN channel & Rician flat fading channel & Rayleigh flat fading channel \\
\hline-4 & $45.0 \%$ & $35.1 \%$ & $31.0 \%$ \\
-3 & $57.0 \%$ & $45.6 \%$ & $37.0 \%$ \\
-2 & $66.2 \%$ & $55.44 \%$ & $46.8 \%$ \\
-1 & $74.2 \%$ & $64.64 \%$ & $54.9 \%$ \\
0 & $81.0 \%$ & $72.64 \%$ & $63.4 \%$ \\
1 & $85.2 \%$ & $78.84 \%$ & $70.8 \%$ \\
2 & $88.4 \%$ & $83.36 \%$ & $76.8 \%$ \\
3 & $91.6 \%$ & $87.16 \%$ & $81.0 \%$ \\
4 & $94.4 \%$ & $89.6 \%$ & $85.1 \%$ \\
5 & $96.6 \%$ & $91.4 \%$ & $87.8 \%$ \\
6 & $98.4 \%$ & $92.76 \%$ & $89.8 \%$ \\
7 & $99.4 \%$ & $93.76 \%$ & $91.2 \%$ \\
8 & $99.91 \%$ & $94.36 \%$ & $91.8 \%$ \\
9 & $100 \%$ & $94.87 \%$ & $92.0 \%$ \\
10 & $100 \%$ & $95 \%$ & $92.0 \%$ \\
\hline
\end{tabular}

TABLE 6: Performance comparison with existing techniques.

\begin{tabular}{|c|c|c|}
\hline Method year, and reference & Features used & $\begin{array}{l}\text { Classification accuracy at } \\
10 \mathrm{~dB} \text { of SNR }\end{array}$ \\
\hline Zero Crossing (1995) [21] & $\begin{array}{l}\text { PDF of cross related } \\
\text { variables }\end{array}$ & $\begin{array}{c}98 \% \\
15 \mathrm{~dB} \text { of SNR }\end{array}$ \\
\hline Hierarchical Architecture (1990) [22] & Spectral features & $90 \%$ \\
\hline $\begin{array}{l}\text { Multilayer Perceptron, Hierarchical SVM + Bees } \\
\text { Algorithm for Optimization (2012) [11] }\end{array}$ & $\begin{array}{l}\text { Spectral features } \\
\text { HOM }\end{array}$ & $\begin{array}{c}97.45 \% \\
\text { (w/o optimization) } \\
99.83 \% \\
\text { (optimization) }\end{array}$ \\
\hline SVM + PSO (2012) [12] & $\begin{array}{c}\text { Spectral features } \\
\text { Statistical features } \\
\text { Wavelets features }\end{array}$ & $98.8 \%$ \\
\hline Artificial Neural Network (2003) [23] & Spectral features & $\begin{array}{c}93 \% \\
8 \mathrm{~dB} \text { of SNR }\end{array}$ \\
\hline Genetic Algorithm based Clustering (2011) [10] & Spectral features & $\begin{array}{c}98.32 \%(\mathrm{GA}) \\
98.12 \%(K \text {-mean })\end{array}$ \\
\hline Hierarchical Architecture (2000) [24] & HOC \& HOM & $96 \%$ \\
\hline Fuzzy based Classifier (2000) [25] & $\begin{array}{c}\text { Kurtosis } \\
\text { phase histogram }\end{array}$ & $\begin{array}{c}90 \% \\
5 \mathrm{~dB} \text { of SNR }\end{array}$ \\
\hline $\begin{array}{l}\text { Multilayer Perceptron Neural Network Recognizer } \\
(2004)[13]\end{array}$ & $\begin{array}{l}\text { Spectral features } \\
\text { cumulants }\end{array}$ & $99.94 \%$ \\
\hline $\begin{array}{l}\text { Binary SVM, Multi SVM GA for Optimization (2010) } \\
\text { [14] }\end{array}$ & HOM \& HOC & $\begin{array}{c}98.5 \% \\
\text { (w/o optimization) } \\
99.36 \% \\
\text { (optimization) } \\
\end{array}$ \\
\hline PSO-SVM based Intelligent Classifier (2013) [17] & HOC & $96 \%$ \\
\hline Gabor Filter (2014) [20] & $\begin{array}{l}\text { Shift, scale, and modulation } \\
\text { parameters }\end{array}$ & $100 \%$ \\
\hline Proposed MGF Network based classifier & $\begin{array}{l}\text { Shift, scale, modulation, } \\
\text { and weights }\end{array}$ & $\begin{array}{c}100 \% \\
(8 \mathrm{~dB} \text { of SNR })\end{array}$ \\
\hline
\end{tabular}

The efficient features extraction from the Gabor filter network easily classifies the considered modulation formats with very low probability of error.

Table 6 shows the classification performance comparison with well-known existing techniques. The classification performance of proposed MGF network based classifier is much better at lower SNRs. The proposed classifier is also capable of classifying $M$-PSK, $M$-QAM, and $M$-FSK [20]. The three features are used to classify $M$-PAM signals.

\section{Conclusion}

On the basis of simulations, it can be concluded that proposed MGF network efficiently classifies the $M$-PAM signals on 
AWGN channel as well as Rician flat fading channel and Rayleigh flat fading channel. The training and testing of proposed MGF network algorithm are done using Delta rule and RLS algorithm which shows the $100 \%$ classification accuracy at $8 \mathrm{~dB}$ of SNR. The classification accuracy is much better when compared with state-of-the-art wellknown techniques. In our future work, we intend to use other biologically inspired computational intelligence algorithms for optimizing the results and higher classification accuracy.

\section{Conflict of Interests}

The authors declare that there is no conflict of interests regarding the publication of this paper.

\section{References}

[1] S. Haykin, "Cognitive radio: brain-empowered wireless communications," IEEE Journal on Selected Areas in Communications, vol. 23, no. 2, pp. 201-220, 2005.

[2] S. A. Ghauri, I. M. Qureshi, A. N. Malik, and T. A. Cheema, "Higher order cummulants based digital modulation recognition scheme," Research Journal of Applied Sciences, Engineering \& Technology, vol. 6, no. 20, pp. 3910-3915, 2013.

[3] P. Panagiotou, A. Anastasopoulos, and A. Polydoros, "Likelihood ratio tests for modulation classification," in Proceedings of the IEEE 21st Century Military Communications Conference (MILCOM '00), vol. 2, pp. 670-674, Los Angeles, Calif, USA, 2000.

[4] O. A. Dobre, A. Abdi, Y. Bar-Ness, and W. Su, "Survey of automatic modulation classification techniques: classical approaches and new trends," IET Communications, vol. 1, no. 2, pp. 137-156, 2007.

[5] F. Wang and X. Wang, "Fast and robust modulation classification via Kolmogorov-Smirnov test," IEEE Transactions on Communications, vol. 58, no. 8, pp. 2324-2332, 2010.

[6] T. Yücek and H. Arslan, "A novel sub-optimum maximumlikelihood modulation classification algorithm for adaptive OFDM systems," in Proceedings of the IEEE Wireless Communications and Networking Conference (WCNC '04), vol. 2, pp. 739-744, March 2004.

[7] W. Wei and J. M. Mendel, "Maximum-likelihood classification for digital amplitude-phase modulations," IEEE Transactions on Communications, vol. 48, no. 2, pp. 189-193, 2000.

[8] W. Su, J. L. Xu, and M. Zhou, "Real-time modulation classification based on maximum likelihood," IEEE Communications Letters, vol. 12, no. 11, pp. 801-803, 2008.

[9] S. A. Ghauri, I. M. Qureshi, A. N. Malik, and T. A. Cheema, "Automatic digital modulation recognition technique using higher order cummulants on faded channels ," Journal of Basic and Applied Scientific Research, vol. 4, no. 3, pp. 1-12, 2014.

[10] A. Ebrahimzadeh and M. H. Hossienzadeh, "A novel method using GA-based clustering and spectral features for modulation classification," in Proceedings of the International Conference on Electrical and Control Engineering (ICECE '11), pp. 4705-4708, IEEE, Yichang, China, September 2011.

[11] A. E. Sherme, "A novel method for automatic modulation recognition," Applied Soft Computing Journal, vol. 12, no. 1, pp. 453-461, 2012.

[12] M. H. Valipour, M. M. Homayounpour, and M. A. Mehralian, "Automatic digital modulation recognition in presence of noise using SVM and PSO," in Proceedings of the 6th International Symposium on Telecommunications (IST '12), pp. 378-382, November 2012.

[13] M. L. D. Wong and A. K. Nandi, "Automatic digital modulation recognition using artificial neural network and genetic algorithm," Signal Processing, vol. 84, no. 2, pp. 351-365, 2004.

[14] A. Ebrhimzadeh, H. Azimi, and H. M. Naeemi, "Classification of communication signals using an optimized classifier and efficient features," The Arabian Journal for Science and Engineering, vol. 35, no. 1, pp. 225-235, 2010.

[15] L. Y. Ling, L. Bing, and Y. C. Yi, "Modulation classification of MQAM signals using particle swarm optimization and subtractive clustering," in Proceedings of the IEEE 10th International Conference on Signal Processing (ICSP '10), pp. 1537-1540, Beijing, China, October 2010.

[16] A. Ebrahimzadeh and R. Ghazalian, "Blind digital modulation classification in software radio using the optimized classifier and feature subset selection," Engineering Applications of Artificial Intelligence, vol. 24, no. 1, pp. 50-59, 2011.

[17] Y. Li, H. Si, M. Liu, and B. Li, "Digital modulation classification in cognitive radio using hybrid particle swarm optimization algorithm-support vector machine," Journal of Computational Information Systems, vol. 9, pp. 3579-3586, 2013.

[18] Z. Zhu, M. Waqar Aslam, and A. K. Nandi, "Genetic algorithm optimized distribution sampling test for M-QAM modulation classification," Signal Processing, vol. 94, no. 1, pp. 264-277, 2014.

[19] Y. Yuan and W. Mei, "Digital modulation classification using multi-layer perceptron and time-frequency features," Journal of Systems Engineering and Electronics, vol. 18, no. 2, pp. 249-254, 2007.

[20] S. A. Ghauri, I. M. Qureshi, T. A. Cheema, and A. N. Malik, "A novel modulation classification approach using Gabor filter network," The Scientific World Journal, vol. 2014, Article ID 643671, 14 pages, 2014.

[21] E. E. Azzouz and A. K. Nandi, "Automatic identification of digital modulation types," Signal Processing, vol. 47, no. 1, pp. 55-69, 1995.

[22] S.-Z. Hsue and S. S. Soliman, "Automatic modulation classification using zero crossing," IEE Proceedings: Radar and Signal Processing, vol. 137, no. 6, pp. 459-464, 1990.

[23] Y. Zhao, G. Ren, X. Wang, Z. Wu, and X. Gu, "Automatic digital modulation recognition using artificial neural networks," in Proceedings of the International Conference on Neural Networks and Signal Processing (ICNNSP '03), pp. 257-260, December 2003.

[24] J. Lopatka and P. Macrej, "Automatic modulation classification using statistical moments and a fuzzy classifier," in Proceedings of the 5th International Conference on Signal Processing Proceedings (ICSP '00), vol. 3, pp. 1500-1506, IEEE, Beijing, China, 2000.

[25] A. Swami and B. M. Sadler, "Hierarchical digital modulation classification using cumulants," IEEE Transactions on Communications, vol. 48, no. 3, pp. 416-429, 2000.

[26] W. M. Waide, "Aircraft landing gear with integrated extension, retraction, and leveling feature," U.S. Patent 7,942,366, 2011. 


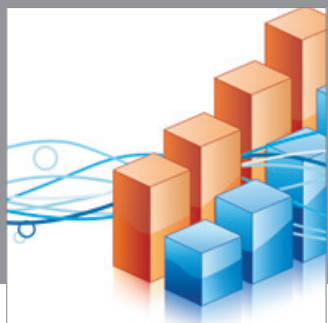

Advances in

Operations Research

mansans

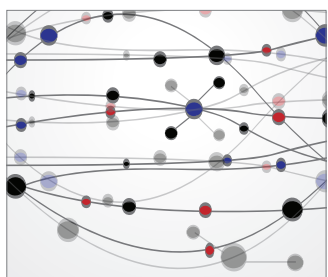

The Scientific World Journal
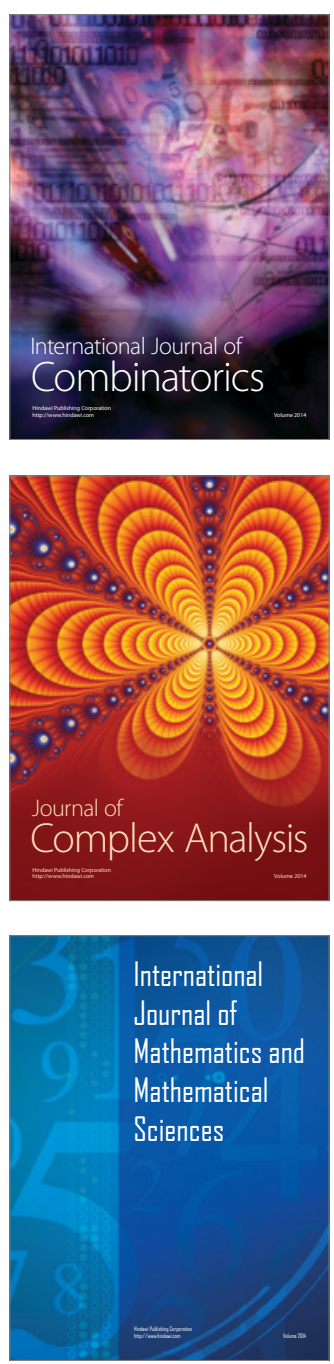
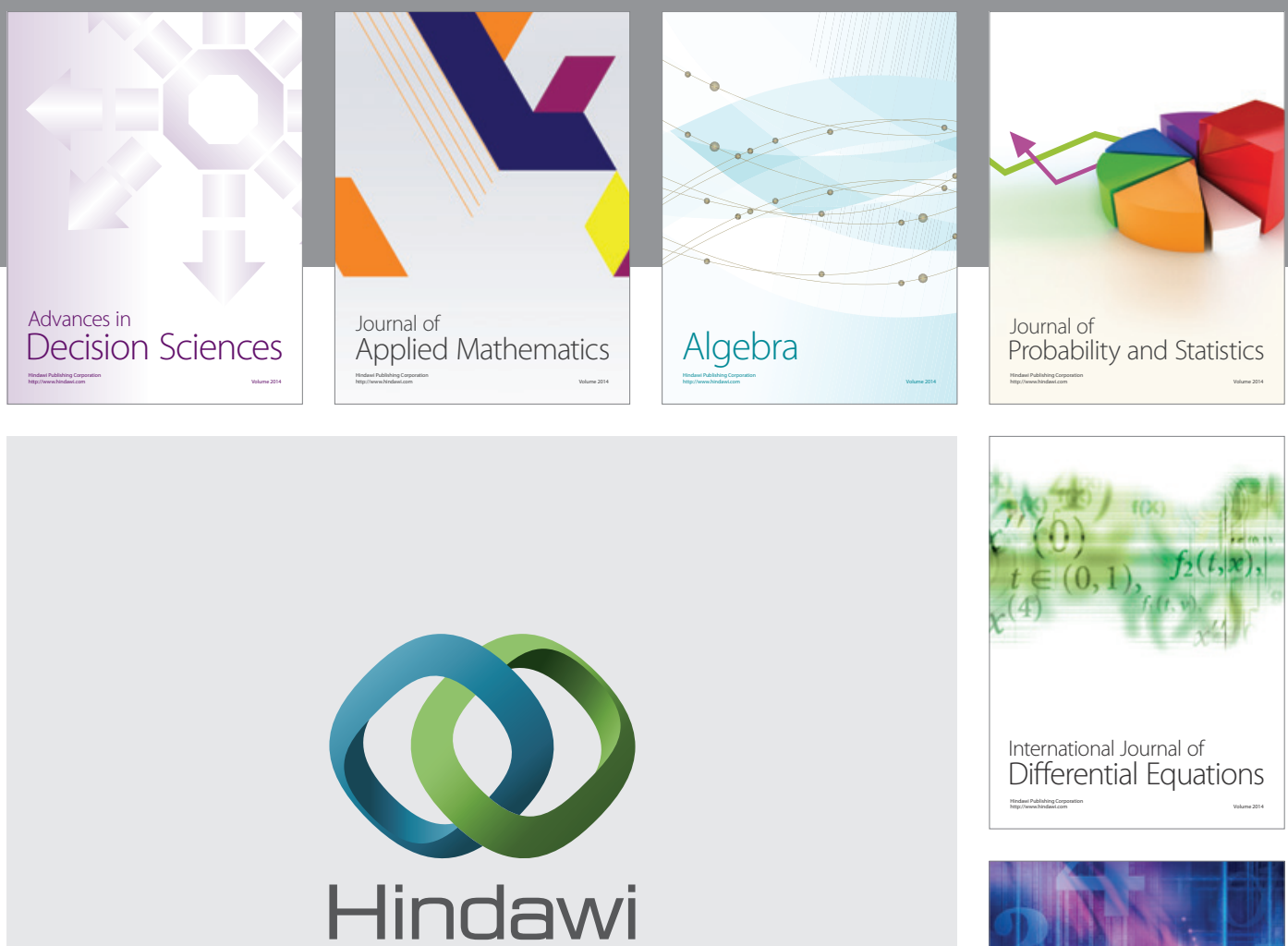

Submit your manuscripts at http://www.hindawi.com
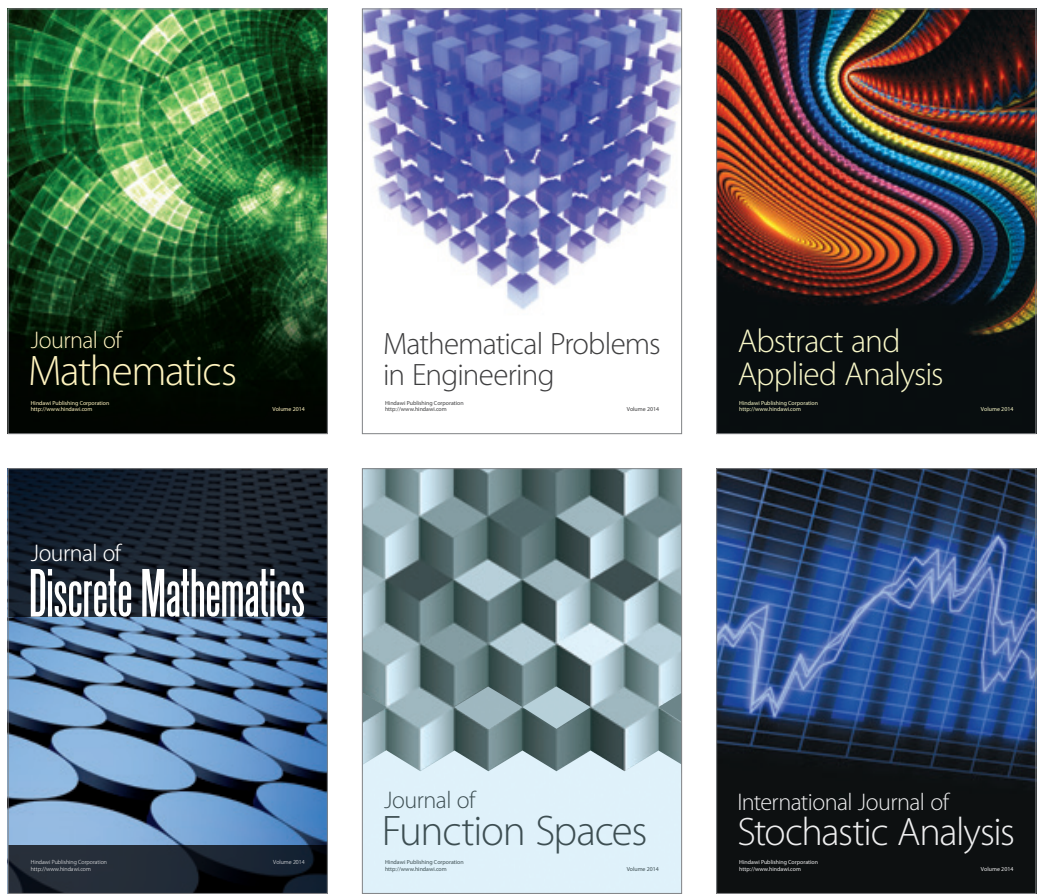

Journal of

Function Spaces

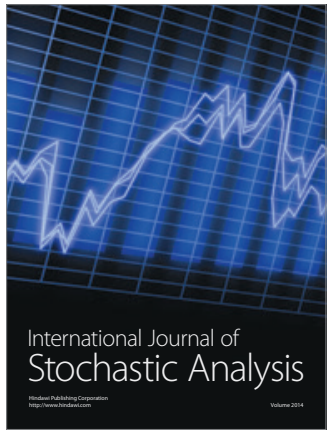

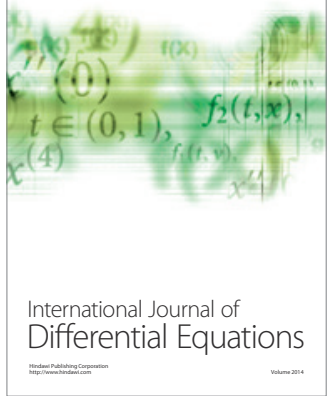
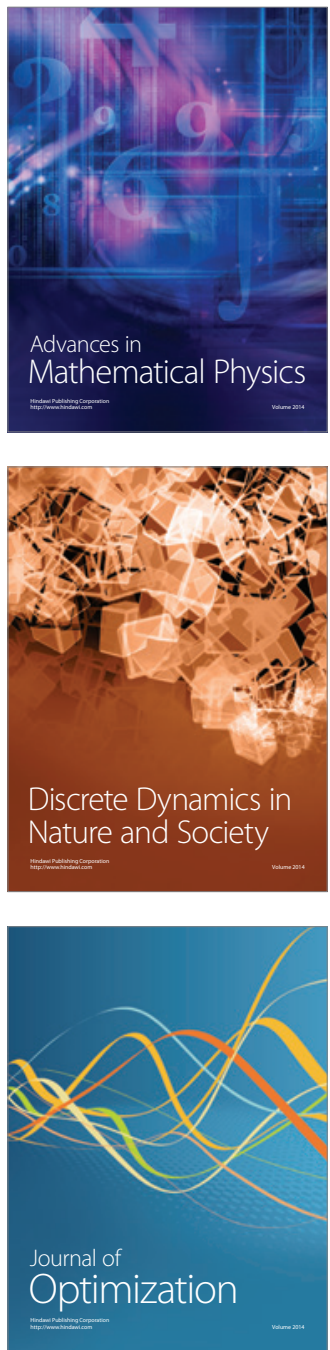